\title{
Measuring Protein Interactions Using Förster Resonance Energy Transfer and Fluorescence Lifetime Imaging Microscopy
}

\author{
Richard N. Day ${ }^{1}$ \\ ${ }^{1}$ Department of Cellular and Integrative Physiology, Indiana University School of Medicine, 635 Barnhill Dr., \\ Indianapolis, IN 46202 USA
}

The development of non-invasive quantitative imaging techniques to visualize dynamic protein behavior inside living cells has become increasingly important for biomedical research. Coupled with the recent improvements in the photophysical qualities of the many different genetically encoded fluorescent proteins (FPs), these imaging approaches enable the measurement of protein behaviors and interactions inside living cells. Here, the method of fluorescence lifetime imaging microscopy (FLIM) is described, and its use to monitor Förster resonance energy transfer (FRET) between labeled proteins inside living cells is demonstrated.

The fluorescence lifetime is the average time that a fluorophore spends in the excited state before returning to the ground state; an event generally coupled to the emission of a photon. Detecting changes in the fluorescence lifetime of a fluorophore can be used to measure events in the surrounding environment. For example, energy transfer is a quenching process that affects the excited state of a fluorophore. When proteins labeled with donor and acceptor FPs interact and FRET occurs, there is a shortening of the donor fluorescence lifetime, and this can be accurately quantified using FLIM [1].

The frequency domain (FD) FLIM approach described here uses a laser modulated at high frequencies (1- 200 $\mathrm{MHz})$ to excite the fluorophores. Because of the lifetime of the excited state, there is a phase delay $(\Phi)$ and a change in the modulation (M) of the emission signal relative to the excitation waveform (Fig. $1 \mathrm{~A}$ ). The emission signals are compared to the excitation waveform, and fluorescence lifetimes are directly determined from both the $\Phi$ and the $M$ measured at the different modulation frequencies $(\omega)$. Phasor plots are used to represent the data, and do not require fitting algorithms to determine the different lifetime components that result from FRET (Fig. 1 B, C).

Here, the FLIM imaging method is demonstrated in living cells expressing 'FRET standard' proteins. The FRET standard approach provides a straightforward method to verify both the biological model, and the imaging system used for the measurement of FRET [2]. The original FRET standard fusion proteins consist of monomeric (m)Cerulean directly coupled to mVenus through linkers of different lengths. However, since mCerulean has more than one fluorescence lifetime (Table 1), we replaced it with the improved variants of mCerulean, mCerulean3 [3] or mTurquoise [4]. These newer variants have reduced photoswitching behavior and improved photostability compared to mCerulean, and both have a single component lifetime (Table 1). Importantly, the FRET standards also serve to validate measurements obtained with FRET-based biosensor probes that are designed to monitor intracellular signaling events.

The composite phasor plot in Figure 1C compares the unquenched and quenched donor lifetimes for the different Cerulean3 FRET standard fusion proteins expressed in living cells. The intensity images obtained from each of the cells expressing the different FRET standard fusion proteins are shown in the right panels (i-iii), with the region of interest (ROI) that was used to determine the average lifetime indicated. For the cell expressing Cerulean3-5aa-Amber (Fig. 1C i), the unquenched donor lifetime was $3.96 \mathrm{~ns}$, and was best fit as a single-component decay. The donor lifetime for the low FRET efficiency standard, Cerulean3-TRAF-Venus (Fig. 1C ii) was $3.68 \mathrm{ns,}$ corresponding to an FRET efficiency (EFRET) of 7\%. In contrast, the lifetime distribution for the cell expressing Cerulean3-5aa-Venus (Fig. 1C iii) clearly shows the quenched donor lifetime for this high FRET standard, and falls inside the semicircle, indicative of the multi-component lifetime expected with FRET. 
When analyzing the multi-exponential lifetimes that result from the linked FRET standard protein, the energy transfer efficiency is determined using the amplitude-weighted lifetime. The amplitude-weighted lifetime for Cerulean3-5aa-Venus is $2.36 \mathrm{~ns}$, which corresponds to an $\mathrm{E}_{\mathrm{FRET}}$ of $40 \%$.

Our recent studies applying the FD FLIM method to the detection of the homologous and heterologous protein interactions mediated by the basic region leucine zipper (BZip) transcription factor $\mathrm{C} / \mathrm{EBP} \alpha$ will be described [5].

\section{References:}

[1] R.N. Day, Methods, (2013)http://dx.doi.org/10.1016/j.ymeth.2013.06.017

[2] S.V. Koushik, et al., Biophys J, 91 (2006) L99-L101.

[3] M.L. Markwardt, et al., PloS one, 6 (2011) e17896.

[4] J. Goedhart, et al., Nat Methods, 7 (2010) 137-139.

[5] A.P. Seigel et al., J Biomed Optics, 18 (2013) 25002.
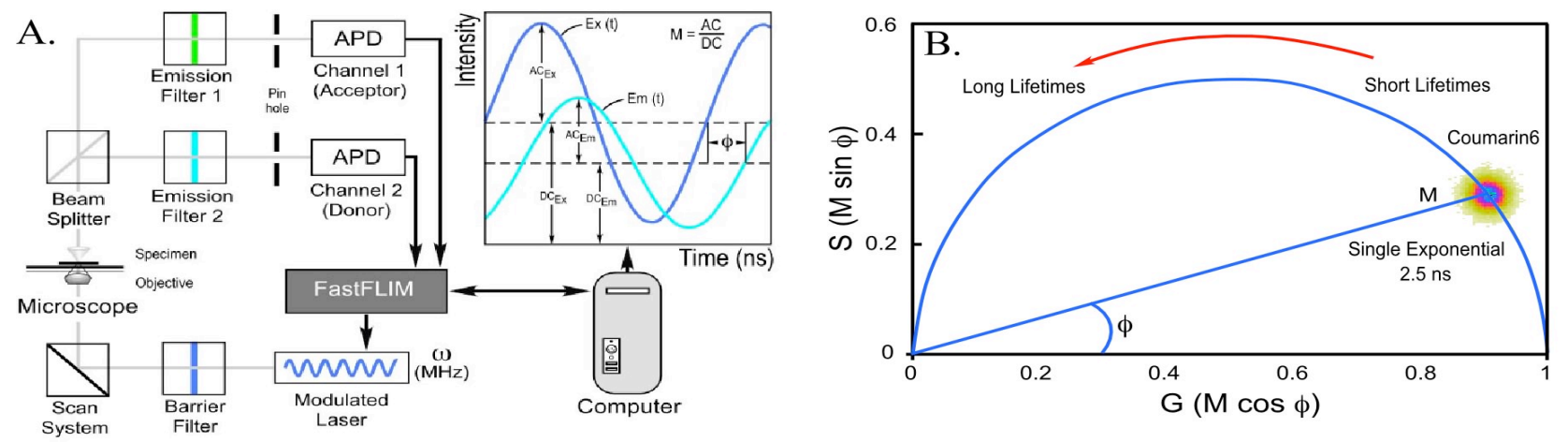

Table 1. FD FLIM analysis of the variants of Cerulean.

\begin{tabular}{|c|c|c|c|}
\hline $\begin{array}{l}\text { Fluorescent } \\
\text { Protein }\end{array}$ & $\begin{array}{c}\text { 2-component lifetime } \\
\text { (Fraction) }\end{array}$ & $\begin{array}{l}\text { Tau(f) } \\
( \pm \text { SD })\end{array}$ & $\begin{array}{c}\mathrm{X}^{20} \\
( \pm \mathrm{SD})\end{array}$ \\
\hline Cerulean & $\begin{array}{l}2.2 \mathrm{~ns}(0.66) \\
4.6 \mathrm{~ns}(0.34)\end{array}$ & $3.0 \pm 0.05$ & $1.1 \pm 0.4$ \\
\hline Cerulean3 & $3.9 \mathrm{~ns}(0.99)$ & $3.9 \pm 0.06$ & $5.7 \pm 2.5$ \\
\hline Turquoise & $3.9 \mathrm{~ns}(0.99)$ & $3.9 \pm 0.06$ & $3.6 \pm 2.0$ \\
\hline Turquoise2 & $4.1 \mathrm{~ns}(0.99)$ & $4.1 \pm 0.04$ & $3.2 \pm 1.6$ \\
\hline
\end{tabular}

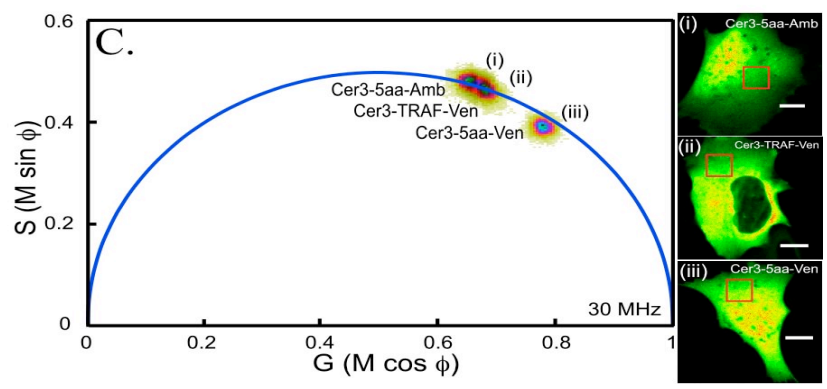

Figure 1. (A) A $440 \mathrm{~nm}$ diode laser modulated at a fundamental frequency of $10 \mathrm{MHz}$ (with as many as 13 harmonic frequencies) is used for the FD FLIM measurements of proteins labeled with the cyan and yellow FPs. The phase delays $(\phi)$ and modulation ratios $(\mathrm{M})$ of the emission relative to the excitation are measured at each pixel of an image for each frequency. (B) The phasor plot of the lifetime distribution for Coumarin 6 shows the simple geometric representation, where the emission signal at each pixel is represented as a vector with length $\mathrm{M}$ and angle $\phi$. (C) The phasor plot analysis of the lifetime distributions for the three different Cerulean3 FRET standards produced in living cells. The corresponding intensity images and ROI are shown in the right panels; the calibration bar is $10 \mu \mathrm{m}$. 